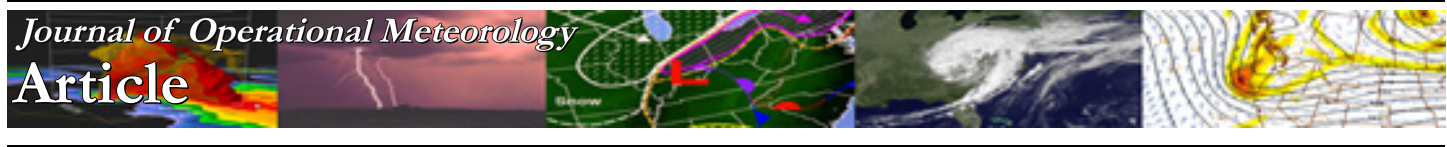

\title{
Early Operational Successes of the University of Louisiana Monroe's Polarimetric S-band Doppler Radar
}

\author{
TODD A. MURPHY \\ University of Louisiana Monroe, Monroe, Louisiana \\ CYNTHIA PALMER \\ NOAA/National Weather Service, San Francisco Bay Area, California ${ }^{1}$ \\ CHAD ENTREMONT \& JAMES D. LAMB \\ NOAA/National Weather Service, Jackson, Mississippi
}

(Manuscript received 1 October 2018; review completed 22 May 2019)

\begin{abstract}
In October 2016, the University of Louisiana Monroe (ULM) began operating a polarimetric S-band Doppler weather radar to help close the low-level radar coverage gap across northern Louisiana by increasing the quantity of data sampled below 3.0 km AGL. Data are delivered in near-real time to local National Weather Service (NWS) Weather Forecast Offices to help meteorologists accomplish their mission of protecting life and property. The inclusion of ULM radar data into NWS operations has led to improved detection of severe and hazardous weather across northern Louisiana. This paper details how the ULM radar has been incorporated into NWS operations, the improvement in operational radar coverage, and the challenges of using a non-NWS radar in the NWS operational setting.
\end{abstract}

\section{Introduction}

The United States national Next Generation Weather Radar (NEXRAD) network remains one of the most important tools available to National Weather Service (NWS) meteorologists for nowcasting and detection of hazardous meteorological phenomena. It also is one of the most advanced national radar networks in the world. However, significant gaps in radar coverage are present throughout the network due to terrain blockage and the large spacing $(200-300 \mathrm{~km})$ between radars (Fig. 1; Maddox et al. 2002). Additionally, the spatial $\left(\sim 1^{\circ}\right.$ beamwidth) and temporal (nominal updates every 4-5 min) resolution of NEXRAD data can limit its use for some applications (e.g., Chandrasekar et al. 2009). However, recent improvements to the NEXRAD system have enabled a lower effective beamwidth $\left(0.5^{\circ}\right)$ through implementation of super-resolution data (Torres and Curtis 2007) and faster volume updates in certain cases through Automated Volume Scan
Evaluation and Termination (AVSET; Chrisman 2009) and Supplemental Adaptive Intravolume Low-Level Scan (SAILS; Crum et al. 2013) options. There have been attempts to investigate the utilization of lowercost radar systems (e.g., X-band radars) to close some of these radar gaps both in the United States and abroad (Chandrasekar et al. 2009; Gourley et al. 2009; Beck and Bousquet 2013; Campbell and Steenburgh 2014), with the Collaborative Adaptive Sensing of the Atmosphere (CASA; McLaughlin et al. 2009) network being the most well-known operational gap-filling network.

One such coverage gap in the NEXRAD network occurs over northeastern Louisiana, southeastern Arkansas, and parts of far western Mississippi (the "ArkLaMiss" region), where the nearest radars are located in Shreveport, Louisiana (KSHV), Brandon, Mississippi (KDGX), and Fort Polk, Louisiana (KPOE),

1 Formerly NOAA/National Weather Service, Shreveport, Louisiana 


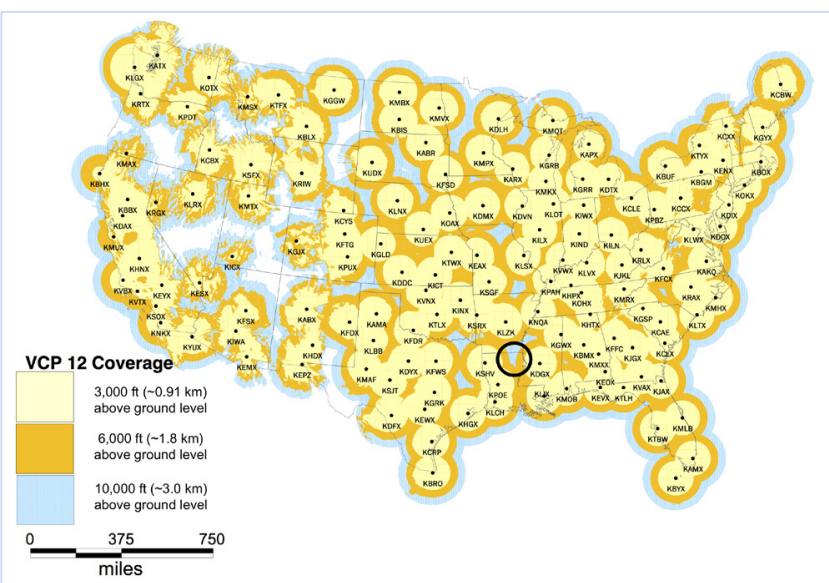

Figure 1. NEXRAD coverage below $\sim 3.0 \mathrm{~km}$ AGL (10 $000 \mathrm{ft}$ AGL) assuming center beam height under standard refractive conditions for the continental United States. The black circle represents the approximate location of the ArkLaMiss and the region served by KULM. Adapted from the Radar Operations Center (2017). Click image for an external version; this applies to all figures hereafter.

and are $>170 \mathrm{~km}$ away from Monroe, Louisiana, the principal city of the region. The distance of these radars from the ArkLaMiss leaves a low-level coverage gap where the minimum beam height (assuming standard atmospheric refraction) is approximately 1.8 to $3.0 \mathrm{~km}$ AGL (6,000 to $10000 \mathrm{ft}$ AGL) (Fig. 1). Such a lowlevel coverage gap presents operational challenges for the local NWS Weather Forecast Offices (WFO) during severe and hazardous weather events. For example, shallow circulations common with quasi-linear convective systems (QLCS) are missed, and typically, only the strongest tornadoes (EF2 or greater) will loft debris to heights detectable above the coverage gap (Gibbs 2016). Strong straight-line, near-surface winds could also be missed.

In an effort to improve severe and hazardous weather detection in the ArkLaMiss, the University of Louisiana Monroe (ULM) began operating a polarimetric S-band Doppler weather radar (hereafter referred to as "KULM") in October 2016. KULM has been successfully incorporated into NWS operations and has been extensively used by WFOs in Shreveport, Louisiana (SHV) and Jackson, Mississippi (JAN) for warning decisions. Improvements in coverage, warning performance, and comparisons with the nearest WSR88D are shown for three high-impact events that occurred in the ArkLaMiss during 2017 and 2018. To provide an objective measure of improvement, simulated tornado probabilities and intensities (Thompson et al. 2017; Cohen et al. 2018) were calculated and compared for each radar. Although KULM has led to significant improvements in radar coverage, a gap-filling, nonNWS radar is not without limitations and challenges within the operational NWS environment. Best practice recommendations for other NWS offices who may have the ability to incorporate gap-filling radars into their operations are given.

\section{The ULM S-band radar}

In 2012, ULM was awarded an approximately $\$ 3$ million grant by the Louisiana Governor's Office of Homeland Security and Emergency Preparedness (GOHSEP) to construct a radar to reduce the ArkLaMiss coverage gap. ULM contracted with Enterprise Electronics Corporation (EEC) to construct a radar in Monroe, Louisiana (KULM) that is nearly equivalent to the Weather Surveillance Radar - 1988 Doppler (WSR-88D) that comprises the NEXRAD network. The primary purpose of KULM is to improve hazard mitigation across the ArkLaMiss, and this is accomplished by providing the data in near real-time to local NWS WFOs to support their mission of protecting life and property. NWS WFOs SHV and JAN began receiving real-time volumetric KULM data in February 2017; data access for NWS WFO Little Rock, Arkansas (LZK) and the Storm Prediction Center (SPC) followed in April 2017.

In addition to the radar's primary purpose, it also serves as an important teaching and research tool for ULM's Department of Atmospheric Science (ATMS), which offers the only Bachelor of Science degree in atmospheric science or meteorology in Louisiana. As of the writing of this paper, ULM is the only primarily undergraduate institution in the United States which owns and operates a polarimetric S-band radar. The radar supports practical, hands-on learning by allowing undergraduate students to gain valuable experience in radar operations, including testing scan strategies. ULM ATMS has leveraged the radar for additional grants to acquire complementary observing equipment (e.g., microwave radiometer, rawinsonde systems, Doppler wind lidar) that further enhances hazard mitigation, teaching, and research. Anchored by KULM, northern Louisiana served as a second observational domain during the spring 2018 Verification of the Origins of Rotation in Tornadoes Experiment - Southeast 
(VORTEX-SE) field campaign (National Severe Storms Laboratory 2018).

\section{a. Specifications and operations}

The KULM radar was manufactured by EEC and represents their DWSR-8501S SIDPOL radar system. It is considered nearly equivalent to the WSR-88Ds which comprise the NEXRAD network. A summary of the major KULM specifications and its comparison to the WSR-88D are given in Table 1. KULM is a polarimetric S-band Doppler radar with an operating frequency (wavelength) of $2.950 \mathrm{GHz}(10.162 \mathrm{~cm})$ and peak transmit power of $850 \mathrm{~kW}$. KULM operates in a simultaneous transmit/receive mode. The pulse repetition frequency (PRF) is user selectable from 200$1180 \mathrm{~Hz}$ dependent upon the pulse duration selections of $0.5,0.8,1.0$, or $2.0 \mu \mathrm{s}$. The gate spacing is $\leq 250 \mathrm{~m}$, with typical operations set at $125 \mathrm{~m}$. The antenna system, approximately $55 \mathrm{~m} \mathrm{MSL}$, is comprised of a parabolic, prime focus reflector with a diameter of $8.5 \mathrm{~m}$, antenna gain of approximately $46 \mathrm{~dB}$, and horizontal (vertical) half-power beam width equal to $0.93^{\circ}\left(0.91^{\circ}\right)$. Perhaps the biggest deviation from the WSR-88D is the choice in transmitter - KULM uses a magnetron oscillator versus the klystron amplifier used by the WSR-88D. Sireci (2008) outlines the many advantages and disadvantages of the different weather radar transmitter types. Perhaps the biggest differences between the two are cost and coherency. Magnetrons are cheaper, but lack the phase stability of klystron transmitters.

KULM is located at $32.529392^{\circ},-92.012115^{\circ}$, approximately $3 \mathrm{~km}$ northeast of the Monroe Regional Airport (MLU), and $6 \mathrm{~km}$ due east of the ULM campus. Figure 2 shows KULM shortly after it became operational. KULM user control and data transmission are accomplished through a 10Gbps fiber line from the radar site to ULM's campus. Power backup is provided by a natural gas generator. The radar is operated by faculty and students at ULM in both classroom and research environments.

To meet the objectives of the original GOHSEP hazard mitigation grant, KULM operates continuously. Because it is not a NWS radar, scan strategies are not bound by the conventional NEXRAD volume coverage patterns (VCP) (Radar Operations Center 2015; Federal Handbook 2017) but are instead more adaptable and flexible for the given situation. KULM is able to perform both sector and range height indicator (RHI) scans, in addition to the typical plan position indicator

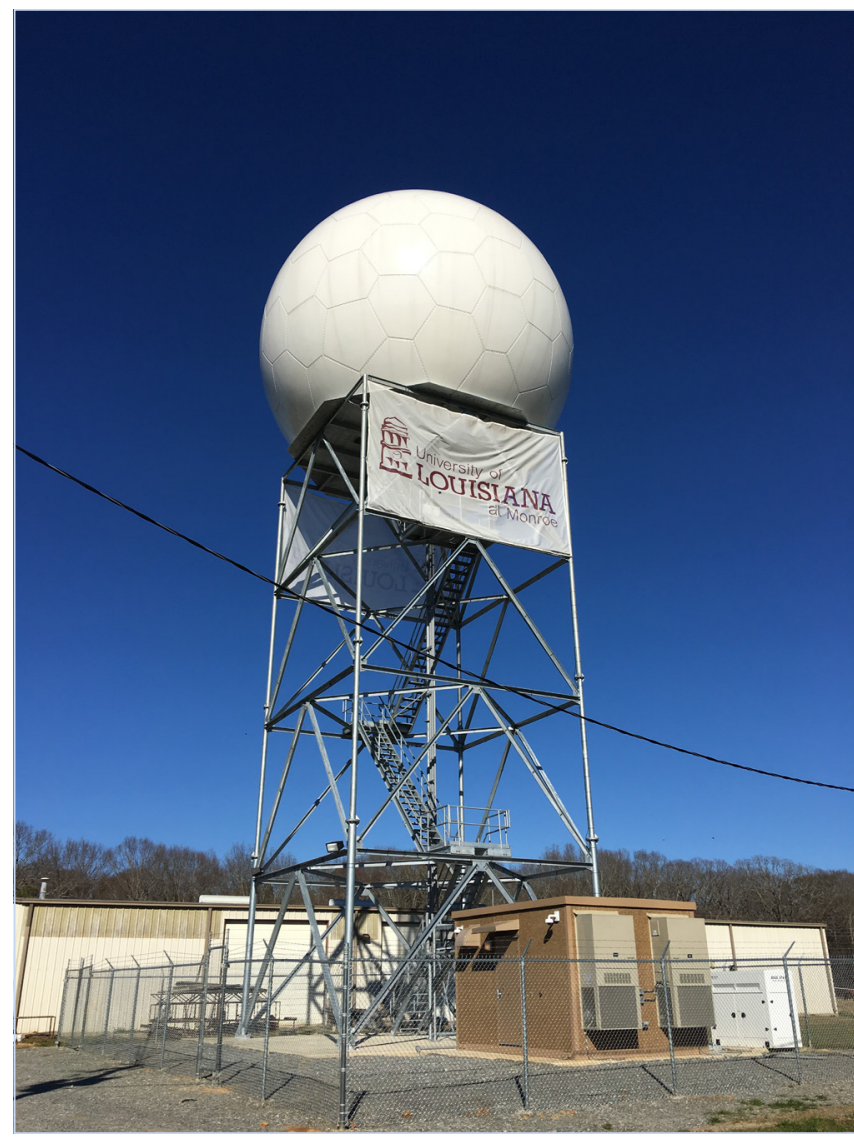

Figure 2. The KULM radar located behind the ULM Visual and Performing Arts annex building, approximately $6 \mathrm{~km}$ due east of ULM's main campus.

(PPI) scans. In that sense, KULM can be considered an operational research radar. For example, to facilitate fast low-level updates, the most common scan strategy during severe and hazardous weather consists of five elevation angles $\left(0.7,1.8,3.1,4.0\right.$, and $\left.5.1^{\circ}\right)$ completing in $\sim 90 \mathrm{~s}$. However, scanning also attempts to mimic NEXRAD VCPs if requested. For example, scan strategies that are similar to VCPs 35 (clear air), 215 (general precipitation), and 12/212 (fast evolving, convective) are available. There also are plans to test novel scan strategies with WFO SHV and JAN during various weather scenarios. The operational KULM data are archived in the CfRadial compliant netcdf format (Dixon et al. 2013) and include the following radar moments: horizontal reflectivity factor $(Z H)$, radial velocity $\left(V_{R}\right)$, spectrum width $(S W)$, differential reflectivity (ZDR), correlation coefficient $(C C)$, and differential propagation phase $\left(\Phi_{D P}\right)$. Raw data, which include the full suite of available radar moments, are archived when the radar is utilized during specific research projects. 
Table 1. Major Specifications of KULM versus the WSR-88D

\begin{tabular}{|l|c|c|}
\hline & KULM & WSR-88D \\
\hline Transmitter Type & Magnetron & Klystron \\
\hline $\begin{array}{l}\text { Frequency } \\
\text { (Wavelength) }\end{array}$ & $\begin{array}{c}2.950 \mathrm{GHz} \\
(10.162 \mathrm{~cm})\end{array}$ & $\begin{array}{c}2.7-3.0 \mathrm{GHz} \\
(\sim 10.71 \mathrm{~cm})\end{array}$ \\
\hline Peak Power & $850 \mathrm{~kW}$ & $700 \mathrm{~kW}$ \\
\hline Pulse Width & $0.5,0.8,1.0, \& 2.0 \mu \mathrm{s}$ & $1.57 \& 4.71 \mu \mathrm{s}$ \\
\hline Antenna Diameter & $8.5 \mathrm{~m}(28 \mathrm{ft})$ & $8.5 \mathrm{~m}(28 \mathrm{ft})$ \\
\hline Beamwidth & $0.93^{\circ}$ & $1^{\circ}$ \\
\hline Gate Spacing & $\leq 250 \mathrm{~m}$ & $250 \mathrm{~m}$ \\
\hline Sensitivity & $-20 \mathrm{dBZ} @ 30 \mathrm{~km} \mathrm{range}$ & $-23 \mathrm{dBZ} \mathrm{@} 25 \mathrm{~km} \mathrm{range}$ \\
\hline Minimum Detectable Signal & $-114 \mathrm{dBm}$ & $-118 \mathrm{dBm}$ \\
\hline Maximum Antenna Rotation Rate & $36^{\circ} / \mathrm{s}$ & $30 \% / \mathrm{s}$ \\
\hline
\end{tabular}

To facilitate data sharing with the NWS, the operational CfRadial data are converted to the NEXRAD level-2, msg-31 format using a locally modified version of the NCAR Radx C++ libraries (Heistermann et al. 2015). NWS real-time access is provided via an internal ULM polling site for the Gibson Ridge (GR) Level 2 Analyst software (www.grlevelx.com/ gr2analyst 2/). These data are publicly available via the Iowa Environmental Mesonet (IEM) NEXRAD level-2 polling site (www.mesonet-nexrad.agron.iastate.edu/ level2/raw/). Live images of $Z_{H}$ and $V_{R}$ at the lowest elevation are generated using the Python ARM Radar Toolkit (Py-ART; Helmus and Collis 2016) and are available via www.wxdata.geos.ulm.edu/ULMradar/.

\section{b. Coverage improvements}

As previously mentioned, the WSR-88Ds nearest Monroe, Louisiana are $>170 \mathrm{~km}$ away, leaving a gap in NEXRAD coverage with some areas having a lowest beam height $>3.0 \mathrm{~km}$ AGL (Fig. 1). Figure 3 shows 100 $\mathrm{km}$ range rings from the nearest WSR-88Ds (KSHV, KDGX, and KPOE), as well as KULM. It additionally shows a $50 \mathrm{~km}$ range ring from KULM. Assuming standard refraction and a $0.5^{\circ}$ elevation angle, the minimum center beam height is $1.8 \mathrm{~km}$ AGL at 100 $\mathrm{km}$, and $0.8 \mathrm{~km}$ AGL at $50 \mathrm{~km}$. KULM, being nearly equidistant from KSHV and KDGX, effectively removes the low-level coverage gap across the ArkLaMiss. In locations within $50 \mathrm{~km}$ of KULM, where the minimum beam height was previously $2.0-3.0 \mathrm{~km}$ AGL, KULM has improved the low-level coverage to $\leq 0.8 \mathrm{~km}$ AGL.

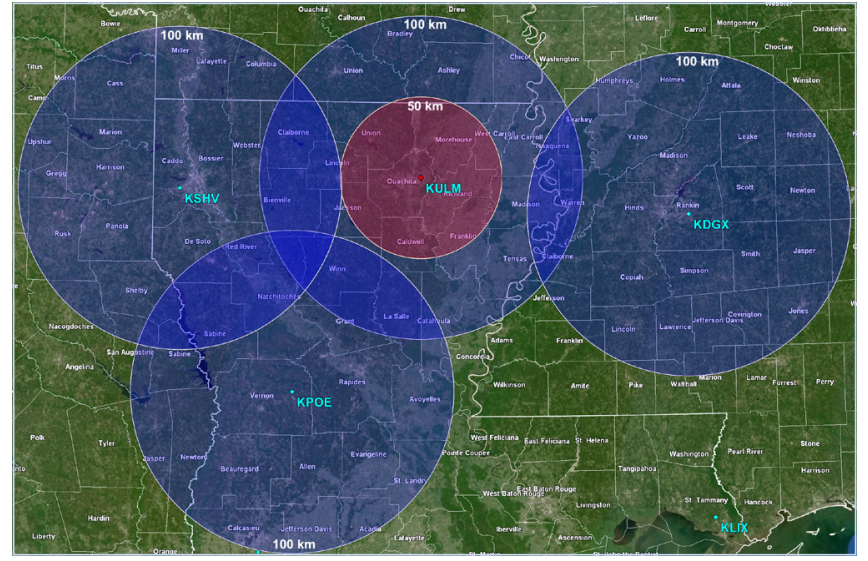

Figure 3. $100 \mathrm{~km}$ range rings from KSHV, KDGX, KPOE, and KULM (blue shaded circles) and a $50 \mathrm{~km}$ range ring from KULM (red shaded circle). At $100 \mathrm{~km}$, minimum center beam height for $0.5^{\circ}$ elevation is $\sim 1.8$ $\mathrm{km}$ AGL. At $50 \mathrm{~km}$, minimum center beam height for $0.5^{\circ}$ elevation is $\sim 0.8 \mathrm{~km}$ AGL.

\section{c. Unresolved issues}

Since beginning operations and providing data to the NWS, several unresolved issues have been noted that have the potential to adversely affect data quality and how the data are used by NWS meteorologists. The KULM data are not currently available via the Advanced Weather Interactive Processing System (AWIPS). The WarnGen program in AWIPS is used exclusively by the NWS for the generation of convective warning products. This software provides a simple method for pinpointing the location of severe weather and projecting storm motion based on radar data displayed within AWIPS. As mentioned above, NWS WFOs use the GRLevel2 
Analyst software to view and analyze KULM data. Because KULM data are not available in AWIPS, it is more difficult for meteorologists to be as precise with storm location and storm motion when using these data to generate warnings.

There also are issues with low-level data gaps. For example, low-level tree blockage adversely affects data up to $\sim 1.5^{\circ}$ elevation between approximate azimuths $323^{\circ}$ and $70^{\circ}$. ULM is working with the landowners to lower or totally remove the trees just north of the radar site. KULM also is located $\sim 1.6 \mathrm{~km}$ due north of the airport surveillance radar (ASR) at the Monroe Regional Airport (MLU), and at its current antenna height, directly interferes with the ASR through power saturation. To remedy this interference, an intentional $10^{\circ}$ sector blank is inserted between azimuths $175^{\circ}$ and $185^{\circ}$ for all elevations $<2.4^{\circ}$. ULM is currently working with the Federal Aviation Administration (FAA) on the ability to remove the sector blank during severe and hazardous weather events, and specifically if a storm of interest is expected to move through the sector blank. However, as of the writing of this paper, FAA approval has not been granted.

\section{Analysis}

\section{a. 2 April 2017}

Supercell thunderstorms developed during the afternoon hours across portions of central and northern Louisiana on 2 April 2017, producing one EF-0, four EF-1, and five EF-2 tornadoes within $100 \mathrm{~km}$ range of KULM (Fig. 4) (National Centers for Environmental Information 2018). Data from KULM were a primary source for warning decisions at NWS Jackson over an approximately 2-h period spanning from 2100-2300 UTC (C. Entremont and J. Lamb, NWS Jackson, 2017, personal communication). The radar was operating in a 3-tilt volume mode $\left(0.7^{\circ}, 1.8^{\circ}\right.$, and $\left.3.1^{\circ}\right)$, providing new updates every $56 \mathrm{~s}$. A radar overview of $Z_{H}$ from KULM is provided as an animation in Fig. 5. The intentional sector blank is immediately apparent to the south of KULM, in addition to range folding in $\mathrm{ZH}$ to the west of the sector blank. Comparisons of KULM and NEXRAD data focused on three tornadoes from the same parent supercell: the Caldwell/Franklin/Richland EF-1, the South Richland EF-1, and the Mangham EF-1 tornadoes (see Fig. 4 for tracks).

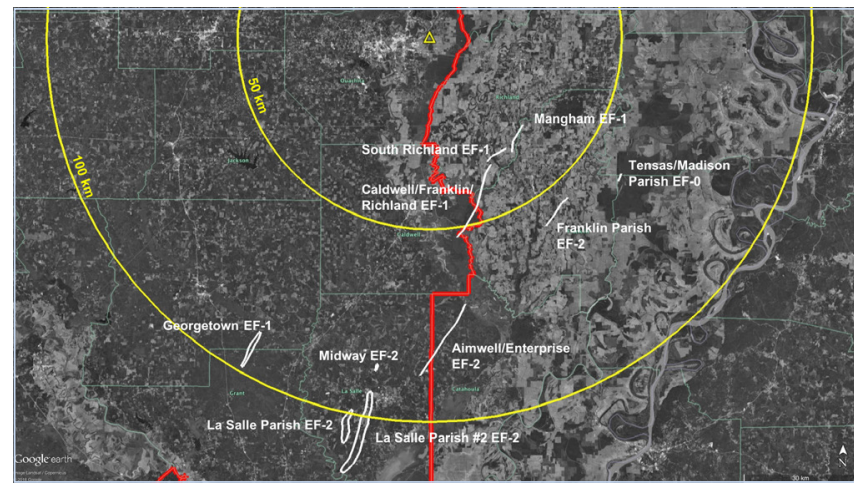

Figure 4. Tornado tracks (white outlines) within 50 and $100 \mathrm{~km}$ range (yellow circles) of KULM (yellow triangle) on 2 April 2017. The red line indicates the county warning area boundary between NWS Shreveport and Jackson.

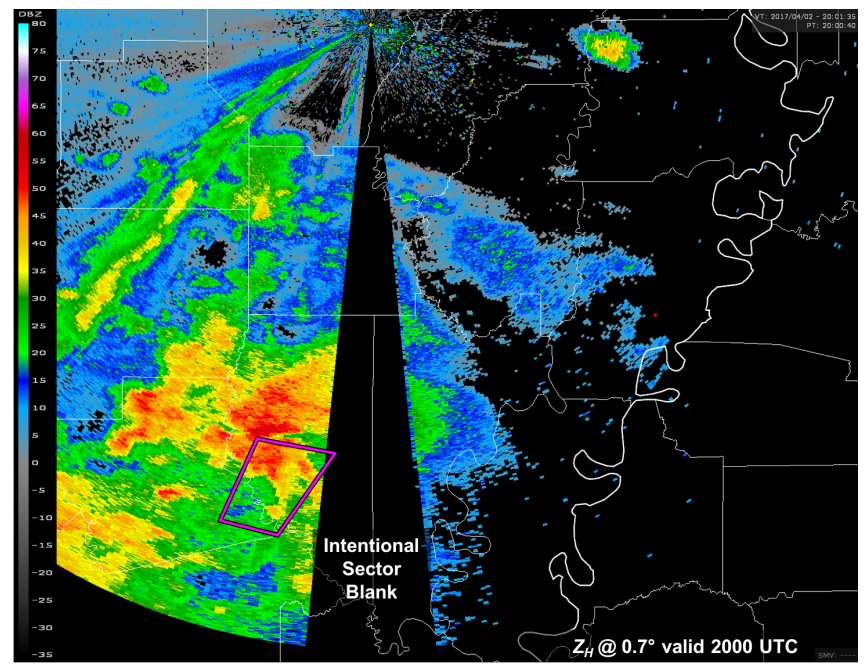

Figure 5. $0.7^{\circ}$ horizontal reflectivity factor $(\mathrm{ZH}$; dBZ) from KULM valid 2000 UTC 2 April 2017. Click the image for an animation of $0.7^{\circ} \mathrm{ZH}$ valid 2000-2343 UTC in 5 min intervals. Tornado warnings (red polygons) and tornado warnings where a tornado has been reported by the public (pink polygons) are overlain.

The Caldwell/Franklin/Richland EF-1 tornado developed at 2117 UTC as the parent supercell's hook echo exited the low-level KULM sector blank. Figure 6a,b shows $Z_{H}$ valid 2112 UTC at $0.7^{\circ}$ and $3.1^{\circ}$ elevation, respectively, of the supercell within the sector blank. WFO SHV issued the first tornado warning on this supercell at 2049 UTC based on midlevel rotation from KPOE and a history of tornado-producing storms. At 2118 UTC, WFO JAN issued a tornado warning prior to the parent supercell crossing into their CWA, effectively continuing the upstream WFO SHV warning. Shortly 


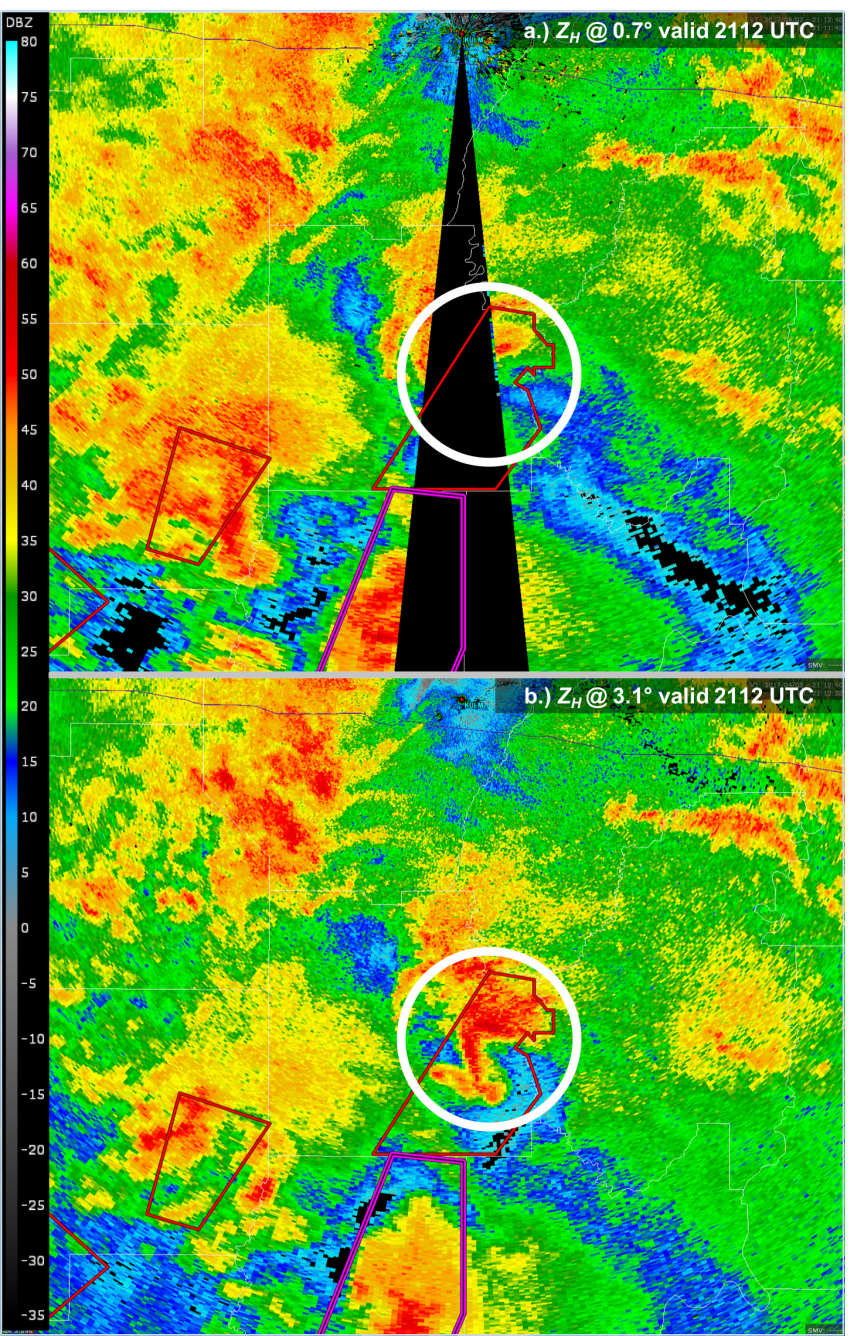

Figure 6. Horizontal reflectivity factor (dBZ) from KULM valid 2112 UTC 2 April 2017 at (a) $0.7^{\circ}$ and (b) $3.1^{\circ}$ elevation angles. The white circle indicates the parent supercell of the Caldwell/Franklin/Richland EF-1 tornado about five min prior to tornadogenesis. Tornado warning polygons are as in Fig. 5.

after exiting the low-level sector blank, a tornadic debris signature (TDS; Rhzhkov et al. 2005; Schultz et al. 2012a,b; Van Den Broeke and Jauernic 2014) was apparent on KULM (Fig. 7a-c). Data from KPOE, the nearest NEXRAD, was ambiguous as to whether a TDS was present (Fig. 7d-f). At its lowest elevation angle $\left(0.7^{\circ}\right)$, KULM sampled the TDS in Fig. 7a-c at $\sim 0.8$ $\mathrm{km}$ AGL; KPOE sampled the same area at $\sim 2.5 \mathrm{~km}$ AGL at $0.4^{\circ}$ (Fig. 7d-f). The KULM data allowed WFO JAN to enhance the wording in their tornado warning to "radar confirmed tornado" at 2123 UTC, which would not have been possible with the NEXRAD data alone.

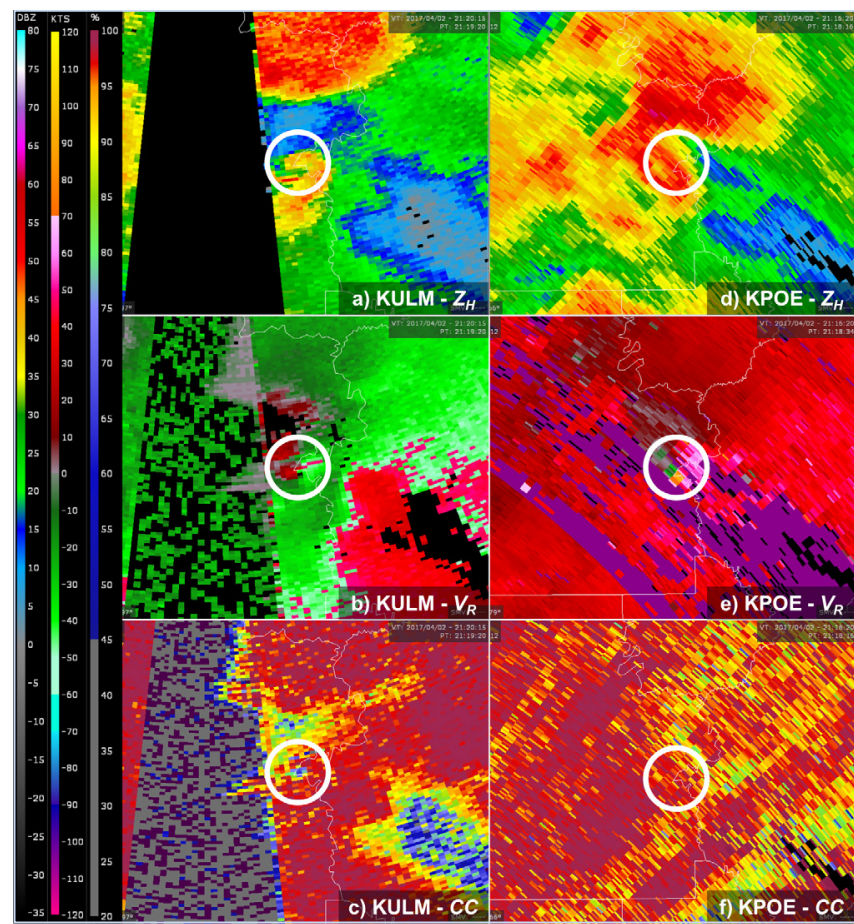

Figure 7. KULM and KPOE comparison for the Caldwell/Franklin/Richland EF-1 tornado near the time of tornadogenesis. Shown for KULM are (a) horizontal reflectivity factor (dBZ), (b) radial velocity (kts), and (c) correlation coefficient at $0.7^{\circ}$ elevation valid 2119 UTC 2 April 2017. Panels (d)-(f) are similar but from KPOE at $0.4^{\circ}$ elevation valid 2118 UTC. White circles indicate the location of the initial TDS observed from KULM. Velocity aliasing is present in (b) ${ }^{2}$. Click the image for an animation of the KULM data valid 2114 2143 UTC in 1 min intervals.

Following Thompson et al. (2017) and Cohen et al. (2018), simulated tornado probabilities and intensities were calculated from each radar dataset using the lowest elevation angle over the tornado's duration. Cohen et al. (2018) determined the best predictors for tornado potential are peak average rotational velocity (VROT; kts), circulation diameter (DIST; n mi), a subjective clear or tight circulation designation (CT), and effectivelayer significant tornado parameter (STP). Similarly, the best predictors for simulated tornado intensity are height of circulation above radar level (ARL; ft), VROT, STP, and whether or not a TDS is present. KULM data

${ }^{2}$ KULM data were stripped of information regarding its Nyquist velocity during data conversion, leading to a failure of the automatic de-aliasing algorithm in GRLevel2. This was corrected in summer 2017. 
with input predictors of ARL $=2310 \mathrm{ft}$, VROT $=44.2$ kts, DIST $=0.2 \mathrm{n} \mathrm{mi,} \mathrm{CT}=1$, TDS $=1$, and STP $\approx$ 4 yielded a simulated tornado probability of $69 \%$ and simulated tornado wind speed of $106 \mathrm{mph}$. The actual estimated peak wind speed based on the NWS survey was $110 \mathrm{mph}$. KPOE data yielded a simulated tornado probability of $37 \%$ and simulated tornado wind speed of $101 \mathrm{mph}$ (input predictors: $\mathrm{ARL}=7000 \mathrm{ft}, \mathrm{VROT}=$ $51.3 \mathrm{kts}, \mathrm{DIST}=1.5 \mathrm{n} \mathrm{mi}, \mathrm{CT}=0, \mathrm{TDS}=0$, and $\mathrm{STP} \approx$ 4). Whereas the potential intensity compared favorably between the radars and the actual tornado intensity, KULM data indicated a much higher probability of tornado occurrence, which should improve tornado warning confidence.

The South Richland EF-1 and Mangham EF-1 tornadoes developed at 2148 and 2149 UTC, respectively. KULM data showed two concurrent TDSs (Fig. 8a-c) for approximately two min, indicating the parent supercell produced two tornadoes at the same time. This was later confirmed by the NWS JAN storm survey. KULM sampled these TDSs at $\sim 0.5 \mathrm{~km}$ AGL. The nearest NEXRAD, KDGX, did not indicate any TDS, as the lowest scan sampled was $\sim 3.0 \mathrm{~km}$ AGL (Fig. 8d-f). In addition, the midlevel rotation on KDGX was displaced north of the actual low-level TDSs from KULM (Fig. 8e) and the observed tornado damage path. The KULM data allowed WFO JAN to extend tornado warnings with greater confidence than NEXRAD data alone would have allowed (C. Entremont and J. Lamb, NWS Jackson, 2017, personal communication). KULM data indicated a simulated tornado probability of $74 \%$ and wind speed of $108 \mathrm{mph}$ (input predictors: ARL = $1,570 \mathrm{ft}$, VROT $=48.1 \mathrm{kts}$, DIST $=0.15 \mathrm{n} \mathrm{mi}, \mathrm{CT}=$ 1 , TDS $=1$, and STP $\approx 4$ ), compared to $32 \%$ and 97 mph from KDGX (input predictors: $\mathrm{ARL}=10500 \mathrm{ft}$, VROT $=40.8 \mathrm{kts}$, DIST $=1.0 \mathrm{n} \mathrm{mi}, \mathrm{CT}=0, \mathrm{TDS}=0$, and STP $\approx 4$ ). The actual estimated peak wind speed based on the NWS survey for both tornadoes was 105 $\mathrm{mph}$. STP for this event was estimated from the SPC Mesoscale Analysis archive (www.spc.noaa.gov/exper/ ma_archive/).

\section{b. 30 April 2017}

An early morning QLCS on 30 April 2017 produced 29 tornadoes in WFO JAN's CWA (National Centers for Environmental Information 2018), including an EF-1 tornado near Oak Ridge, Louisiana at 0944 UTC (Fig. 9). KULM was again operating in a 3-tilt volume mode $\left(0.7^{\circ}, 1.8^{\circ}\right.$, and $\left.3.1^{\circ}\right)$, providing new

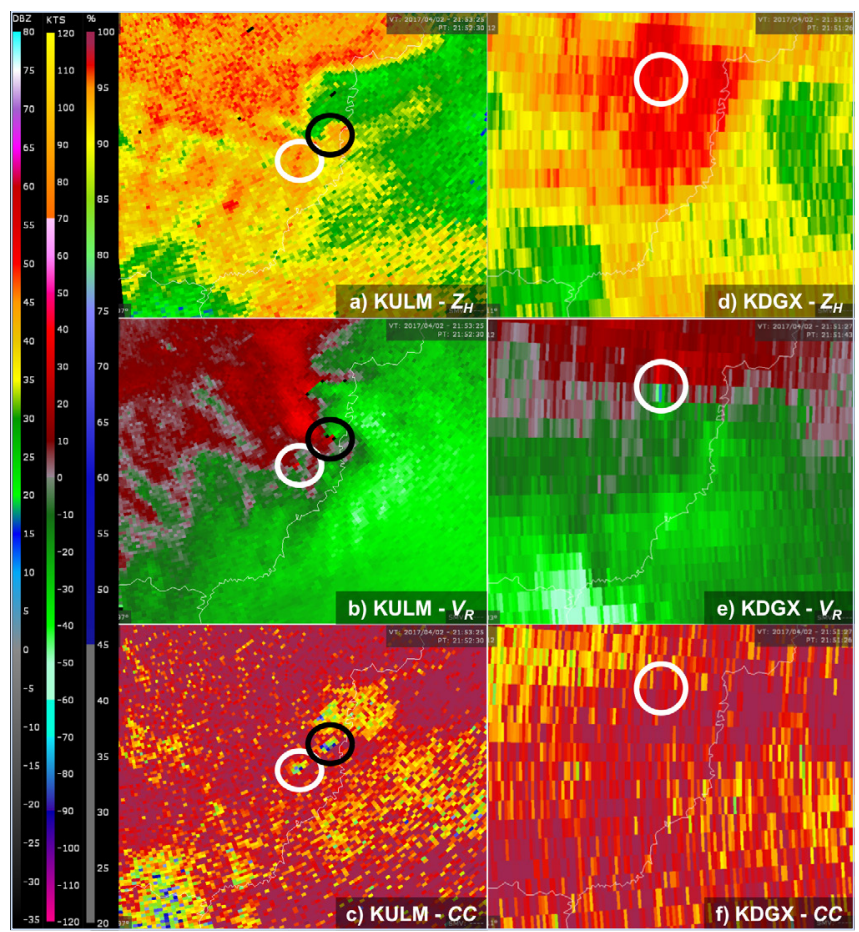

Figure 8. As in Fig. 7 but for KULM at $0.7^{\circ}$ elevation valid 2152 UTC 2 April 2017 and KDGX at elevation $0.5^{\circ}$ valid 2151 UTC 2 April 2017. In panels (a)-(c), the white circle represents the TDS from the South Richland EF-1 tornado and the black circle represents the TDS from the Mangham EF-1 tornado. In panels (d)-(f), the white circle represents the area of strongest rotation on KDGX, which is displaced north of the lowlevel KULM TDSs. Click the image for an animation of the KULM data valid 2145-2201 UTC in $1 \mathrm{~min}$ intervals.

volume scans every $56 \mathrm{~s}$. The region where the tornado developed is within the low-level tree blockage north of KULM, obscuring many of the radar moments at $<1.5^{\circ}$ elevation angle, although $V_{R}$ still provided some usable data because pulse pair phases are not impacted as long as some useful signal is present (Fig. 10). In Fig. 10b, an area of cyclonic rotation in $V_{R}$ is observed even though the remaining moments (Fig. 10a, c) are biased due to tree blockage. A TDS is present at the next elevation angle (1.8 ${ }^{\circ}$; Fig. 10d-f). The TDS ultimately prompted NWS JAN to survey this area, revealing EF-1 tornado damage. No public damage reports were received by the NWS in association with the TDSs near KULM during this event. The corresponding NEXRAD data (Fig. 10g-i) are inconclusive as to what is occurring in this area, and without KULM data, it is likely the EF-1 tornado would have been unwarned and not 


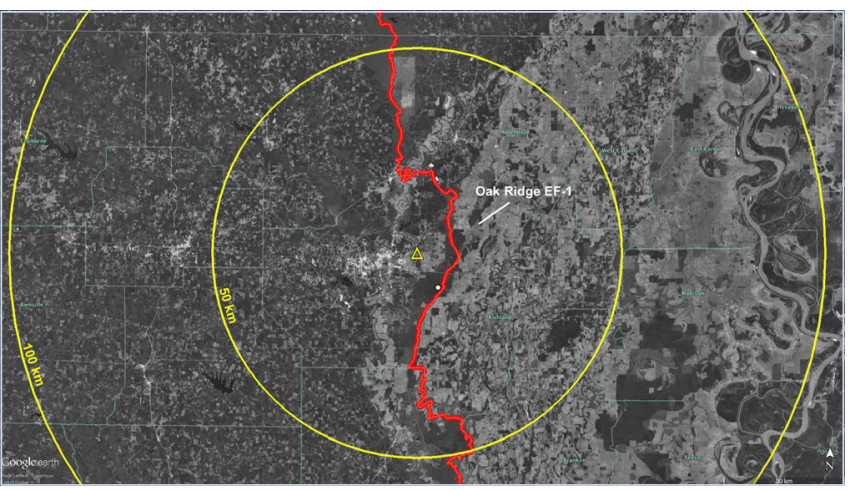

Figure 9. As in Fig. 4 but for 30 April 2017.

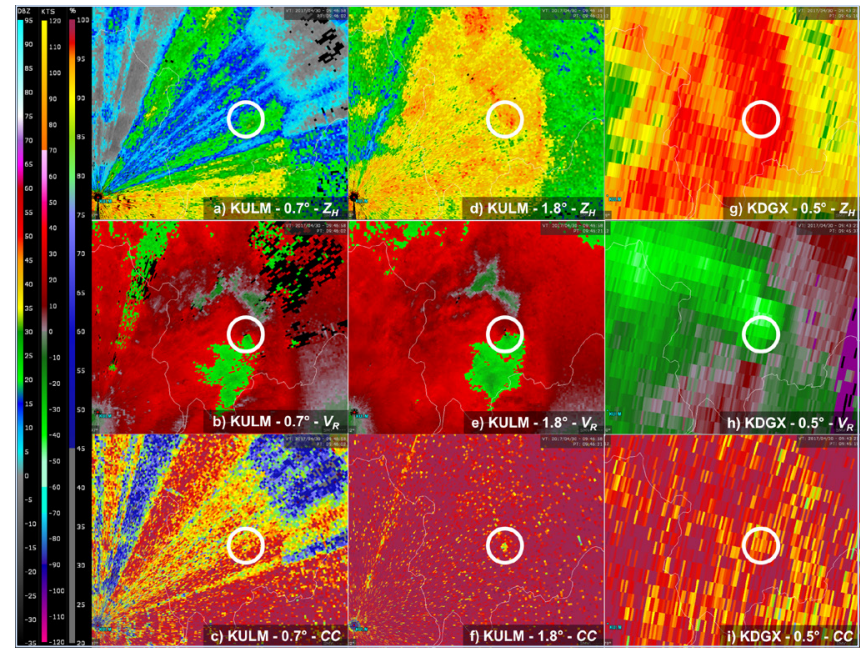

Figure 10. As in Fig. 7 but for KULM at $0.7^{\circ}$ elevation (a)-(c) and $1.8^{\circ}$ elevation (d)-(f) valid 0946 UTC 30 April 2017. Panels (g)-(i) are KDGX at $0.5^{\circ}$ elevation valid 0945 UTC 30 April 2017. The white circle represents the area of rotation and TDS observed on KULM associated with the Oak Ridge EF-1 tornado. The beam height within the circled area is $\sim 0.25 \mathrm{~km}$ AGL in (a)-(c), $\sim 0.6 \mathrm{~km}$ AGL in (d)-(f), and $\sim 3.3 \mathrm{~km}$ AGL in (g)-(i). Velocity aliasing is present in (b) and (e).

surveyed (C. Entremont and J. Lamb, NWS Jackson, 2017, personal communication). Simulated tornado probability and wind speeds were not calculated for this case, given the uncertainty imparted by the low-level tree blockage.

\section{c. 13 April 2018}

A multi-day severe weather episode, with approximately 70 confirmed tornadoes, impacted portions of the southeastern United States on 13-15
April 2018. The bulk of these tornadoes occurred on 13-14 April in Arkansas, Louisiana, and Mississippi. In particular, northern Louisiana and southern Arkansas were impacted by two rounds of severe weather on 13-14 April, including late afternoon/early evening tornadic supercell thunderstorms, and an overnight tornadic QLCS. A total of 23 tornadoes occurred in this region, 17 of those within $100 \mathrm{~km}$ range of KULM and 8 within $50 \mathrm{~km}$ range (Fig. 11) (National Centers for Environmental Information 2018). This event is unique in that operations associated with the VORTEXSE field program occurred in northern Louisiana, leading to an exceptional set of observations for both the supercells and the QLCS. KULM was operating in a 5-tilt volume mode $\left(0.7^{\circ}, 1.8^{\circ}, 3.1^{\circ}, 4.0^{\circ}\right.$, and $\left.5.1^{\circ}\right)$, providing new volume scans every $90 \mathrm{~s}$. Compared herein are KULM and KSHV data associated with the "Calhoun" supercell, which produced the Calhoun EF1, Downsville EF-1, and Sterlington EF-1 tornadoes.

KULM and KSHV data near the genesis time of the Calhoun EF-1 (2334 UTC) tornado are shown in Fig. 12. The initial tornado warning from WFO SHV was issued at 2328 UTC based on "radar indicated rotation" from KULM. A clear hook echo in $Z_{H}$ and broad, cyclonic low-level circulation in $V_{R}$ was observed from KULM (Fig. 12a, b). VROT increased from $25.2 \mathrm{kts}$ to 45.7 kts shortly after the tornado formed; a TDS on KULM was apparent by 2335 UTC. Near this same time, the tornado was visually sighted by the NOAA P-3 research aircraft participating in the VORTEX-SE project (www. mesonet.agron.iastate.edu/wx/afos/201804132340 LSRSHV.png), only the second time the P-3 has sighted a tornado and likely the first time a tornado report from a research aircraft made it into NWS products (C. Ziegler and E. Rasmussen, NOAA NSSL/OU CIMMS, 2018, personal communication). A second tornado, the Downsville EF-1, formed at 2343 UTC. Near the end of

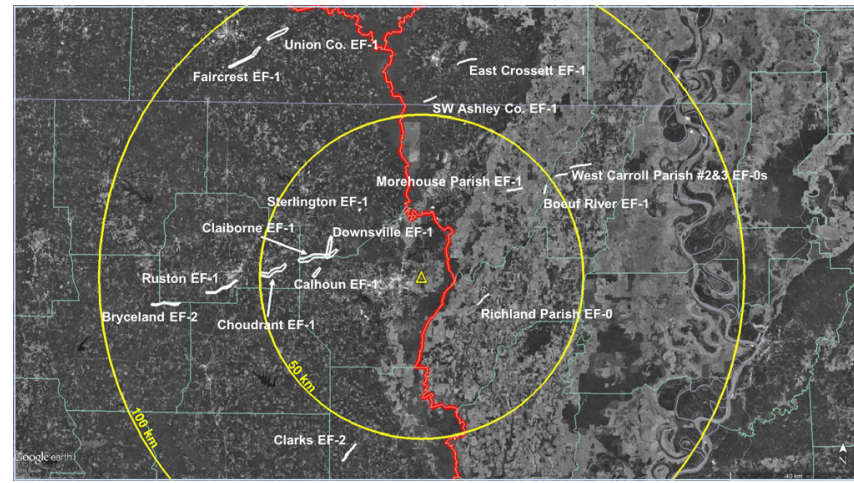

Figure 11. As in Fig. 4 but for 13-14 April 2018. 


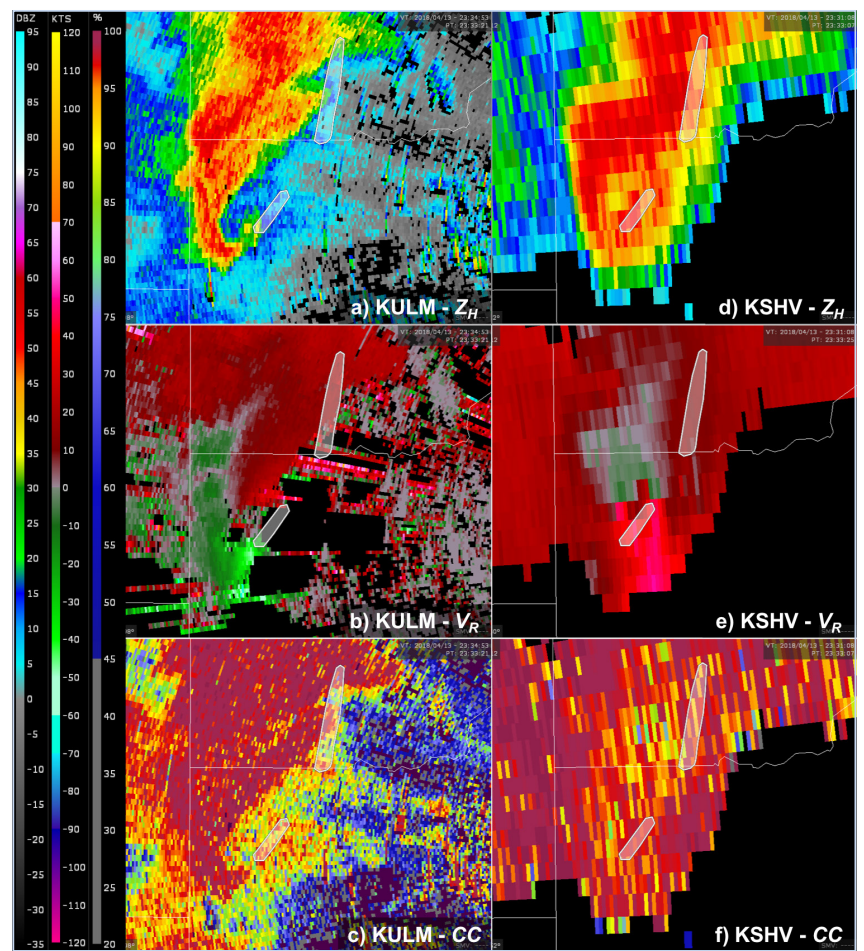

Figure 12. As in Fig. 7 but for KULM at $0.7^{\circ}$ elevation and KSHV at $0.5^{\circ}$ elevation, both valid 2333 UTC 13 April 2018. The white polygons represent the Calhoun EF-1 and Downsville EF-1 tornado tracks. The beam height within the hook echo region of panels (a)-(c) is $\sim 0.4-0.5 \mathrm{~km}$ AGL. For the same region, the KSHV beam height is $\sim 2.5 \mathrm{~km}$ AGL. Click the image for an animation of the KULM data valid 2330-2355 UTC in 90 -s intervals.

this tornado, mesocyclone occlusion is clearly evident on KULM as the hook echo seemingly pulls back into the larger precipitation echo (Fig. 12 animation).

KULM sampled the low-level circulations associated with these two tornadoes at approximately $0.4-0.5 \mathrm{~km}$ AGL. The nearest NEXRAD, KSHV, viewed the storm at approximately $2.5 \mathrm{~km}$ AGL (Fig. 12d-f). From KSHV, modest midlevel rotation (VROT $=26.2 \mathrm{kts}$ ) associated with the mesocyclone was observed; KSHV VROT increased to a maximum of 36.4 kts. KSHV also observed a low-reflectivity region associated with the updraft and bounded weak echo region. The midlevel rotation was displaced north of the low-level circulation and TDS observed via KULM. KULM data indicated a simulated tornado probability of $73 \%$ and wind speed of $106 \mathrm{mph}$ (input predictors: $\mathrm{ARL}=1,395 \mathrm{ft}$, VROT $=47.6 \mathrm{kts}$, DIST $=0.15 \mathrm{n} \mathrm{mi}$, $\mathrm{CT}=1$, $\mathrm{TDS}=1$, and STP $\approx 2.8$ ), compared to $49 \%$ and $91 \mathrm{mph}$ from KSHV (input predictors: $\mathrm{ARL}=7,800 \mathrm{ft}$,
VROT $=36.4 \mathrm{kts}$, DIST $=0.75 \mathrm{n} \mathrm{mi}, \mathrm{CT}=1$, TDS $=$ 0 , and STP $\approx 2.8$ ). STP was estimated from a 23 UTC sounding launched by ULM in Monroe, Louisiana. The actual estimated peak wind speed based on the NWS survey for both tornadoes was $105 \mathrm{mph}$. KSHV data alone were inconclusive regarding the tornado potential of this supercell, and without KULM, WFO SHV would have needed spotter reports to confirm the presence of a tornado. KULM allowed WFO SHV to not only issue more confident tornado warnings, but also refine those warnings with better geographic precision (C. Palmer, NWS Shreveport, 2018, personal communication).

\section{Discussion and conclusion}

Data from non-NEXRAD, gap-filling radars, such as KULM, provide valuable information during the warning-decision process. KULM has become an important tool for NWS WFOs SHV and JAN. It has been incorporated into operations at these offices, helping to improve the NWS mission of protecting life and property. Its data proved invaluable during active spring severe weather seasons in 2017 and 2018 across the ArkLaMiss. At least five additional tornadoes have been warned and surveyed (to date) by WFO JAN that may not have been surveyed otherwise due to a lack of damage reports and inconclusive NEXRAD data. During each of these events TDSs were observed by KULM. This does not imply that NWS warning services are degraded without KULM, but instead, improved radar data lead to improved detection of severe and hazardous weather. A climatology of tornadogenesis points within 100-km of KULM (1950-2014) indicates the region averages approximately eight tornadoes per year. This number is expected to increase, consistent with the tornado reporting trends after implementation of the WSR-88D network in the early 1990s (McCarthy and Schaefer 2004; Coleman and Dixon 2014).

During severe weather operations at WFO SHV and JAN, forecasters now rely on KULM when there is convection over northeastern Louisiana and portions of southeastern Arkansas in the same manner they would rely on other radar sites in the NEXRAD network (C. Entremont, J. Lamb, and C. Palmer, NWS, 2018, personal communication). At WFO JAN, a separate forecaster has been tasked with monitoring KULM and relaying pertinent information to the forecaster issuing warnings during some past more impactful and widespread severe weather events (C. Entremont and J. Lamb, NWS Jackson, 2018, personal communication). 
All workstations used by warning forecasters are equipped with GRLevel2 Analyst software, which forecasters use to monitor KULM data on a real-time basis as they make warning decisions. Because of the closer proximity of KULM, more accurate location information can be added to the NWS warnings in terms of current position of the tornado, along with improved timing for pathcasts (arrival time to towns/cities). This information is extremely valuable to the warning forecaster as well as in communicating more specific location information directly to county officials.

In most severe weather situations, a scanning strategy limited to lower elevation scans has been utilized for KULM. Forecasters have found this to be the most beneficial, as it provides more frequent updates and improves radar coverage for the northeast Louisiana region. Data that would be obtained by scanning at higher elevations are largely already being collected by surrounding radar sites, and thus holds lesser value to NWS meteorologists during active weather events.

An open line of communication between ULM, WFO SHV, and JAN has allowed for improvements in radar operations and data delivery to better support the NWS mission. For example, ULM maintains flexibility in scanning strategy to deliver the best possible information to the WFOs. The WFOs alert ULM personnel if they notice data problems, such as $Z_{H}$ or ZDR calibration issues. However, a non-NEXRAD radar still presents challenges. Data delivery, for example, is dependent on the reliability of the ULM network. KULM volume scans are not available on a "tilt-bytilt" basis, only when a volume completes and after converting to the $88 \mathrm{D}$ compatible format. This can cause a slight time delay in the delivery of volumes. Unavailability in AWIPS also is an inconvenience, as previously noted, though the data are extremely useful even without the ability for AWIPS interrogation. Work is underway to minimize the time delay, and perhaps have the data available via AWIPS. Another important takeaway is that where a radar is built plays a substantial role in terms of data quality. ULM did not have the luxury to select a pristine radar site, but others exploring gap-filling radars should properly survey potential sites to minimize blockage or other issues that adversely impact data quality. Although there is room for additional improvements, the radar is achieving its ultimate goal of improving hazard mitigation across the region.

As the cost of Doppler weather radar systems decrease, there has been an increase in the number of public and private entities acquiring systems of various frequencies. These systems present a unique opportunity for data sharing agreements with the NWS that could be modeled after KULM for gap-filling radars. NWS WFOs should reach out to those radar owners about the possibility of incorporating their data into operations; such data have the potential to enhance warning operations and information shared with partners. If such partnerships are possible, WFOs should work closely with the radar owners to understand the capabilities and limitations of the radar. The NWS should provide radar partners technical support to arrange for data delivery if necessary and also support incorporating non-NEXRAD data into AWIPS.

Acknowledgments: This paper was funded by the ULM Endowed Professorship in Geosciences. Data collection and analysis of the 13 April 2018 case occurred during the VORTEX-SE field project supported by the United States Weather Research Program within NOAA/OAR/OWAQ under contract RA133R18SE0299. We thank the Louisiana GOHSEP for providing funds to support the construction of the ULM radar. Drs. Eric Pani and Anne Case Hanks are acknowledged for the pivotal role they played in securing the original grant to support the radar's construction. Meteorologists at NWS WFO Shreveport and Jackson, and local emergency managers, are acknowledged for their feedback on improving radar operations in support of their missions. Drs. Conrad Ziegler and Erik Rasmussen are acknowledged for information regarding P-3 tornado sightings. Finally, we would like to acknowledge the three anonymous reviewers for providing comments that greatly improved the original manuscript.

\section{REFERENCES}

Beck, J. and O. Bousquet, 2013: Using gap-filling radars in mountainous regions to complement a national radar network: Improvements in multiple-Doppler wind syntheses. J. Appl. Meteor. Climatol., 52, 1836-1850, CrossRef.

Campbell, L. S. and W. J. Steenburgh, 2014: Finescale orographic precipitation variability and gap-filling radar potential in Little Cottonwood Canyon, Utah. Wea. Forecasting, 29, 912-935, CrossRef. 
Chandrasekar, V., Y. Wang, M. Maki, and K. Nakane, 2009: Urban flood monitoring using X-band dual-polarization radar network: Program of the CASA-NIED partnership. Preprints, 34th Conf. on Radar Meteorology, Williamsburg, VA, Amer. Meteor. Soc., P10A.1. [Available online at ams.confex.com/ams/34Radar/ techprogram/paper 156019.htm.]

Chrisman, J. N., 2009: Automated Volume Scan Evaluation and Termination (AVSET): A simple technique to achieve faster volume scan updates. Preprints, 34th Conf. on Radar Meteorology, Williamsburg, VA, Amer. Meteor. Soc., P4.4. [Available online at ams.confex.com/ams/ pdfpapers/155324.pdf.]

Cohen, A. E., J. B. Cohen, R. L. Thompson, and B. T. Smith, 2018: Simulating tornado probability and tornado wind speed based on statistical models. Wea. Forecasting, 33, 1099-1108, CrossRef.

Coleman, T. A., and P. G. Dixon, 2014: An objective analysis of tornado risk in the United States. Wea. Forecasting, 29, 366-376, CrossRef.

Crum, T., S. D. Smith, J. N. Chrisman, R. E. Saffle, R. W. Hall, and R. J. Vogt, 2013: WSR-88D radar projectsUpdate 2013. Proc. 29th Conf. on Environmental Information Processing Technologies, Austin, TX, Amer. Meteor. Soc., 6B.1. [Available online at ams. confex.com/ams/93Annual/webprogram/Paper221461. html.]

Dixon, M., W.-C. Lee, B. Rilling, and C. Burghart, 2013: CfRadial data file format: Proposed CF-compliant netCDF format for moments data for RADAR and LIDAR in radial coordinates. NCAR, $66 \mathrm{pp}$. [Available online at www.eol.ucar.edu/system/files/CfRadialDoc. v1.3.20130701.pdf.]

Federal Handbook, 2017: Federal Meteorological Handbook No. 11, WSR-88D Meteorological Observations, Part C: WSR-88D Products and Algorithms, 394 pp. [Available online at www.ofcm.gov/publications/fmh/ FMH11/fmh11partC.pdf].

Gibbs, J. G., 2016: A skill assessment of techniques for real time diagnosis and short-term prediction of tornado intensity using the WSR-88D. J. Operational Meteor., 4(13), 170-181, CrossRef.

Gourley, J. J., D. P. Jorgensen, S. Y. Matrosov, and Z. L. Flamig, 2009: Evaluation of incremental improvements to quantitative precipitation estimates in complex terrain. J. Hydrometeor., 10, 1507-1520, CrossRef.

Heistermann, M., and co-authors, 2015: The emergence of open-source software for the weather radar community. Bull. Amer. Meteor. Soc., 96, 117-128, CrossRef.

Helmus, J. J., and S. M. Collis, 2016: The Python ARM Radar Toolkit (Py-ART), a library for working with weather radar data in the python programming language. J. Open Research Software, 4(1), 25 pp., CrossRef.
Maddox, R. A., J. Zhang, J. J. Gourley, and K. W. Howard, 2002: Weather radar coverage over the contiguous United States. Wea. Forecasting, 17, 927-934, CrossRef.

McCarthy, D. W., and J. T. Schaefer, 2004: Tornado trends over the past thirty years. Preprints, 14th Conf. on Applied Climatology, Seattle, WA. Amer. Meteor. Soc., 3.4. [Available online at ams.confex.com/ams/84Annual/ techprogram/paper_72089.htm.]

McLaughlin, D., and Coauthors, 2009: Short-wavelength technology and the potential for distributed networks of small radar systems. Bull. Amer. Meteor. Soc., 90, 1797-1817, CrossRef.

National Centers for Environmental Information, 2018: Storm events database. [Available online at www.ncdc. noaa.gov/stormevents/].

National Severe Storms Laboratory, 2018: VORTEX Southeast. [Available online at www.nssl.noaa.gov/ projects/vortexse/].

Radar Operations Center, 2015: WSR-88D Volume Coverage Pattern (VCP) Improvement Initiatives. [Available online at www.roc.noaa.gov/WSR88D/PublicDocs/ New Technology/New VCP Paradigm_Public Oct 2015.pdf

Radar Operations Center, 2017: NEXRAD and TDWR Radar Locations. Accessed 25 May 2018, www.roc.noaa.gov/ WSR88D/maps.aspx.

Ryzhkov, A. V., T. J. Schuur, D. W. Burgess, and D. S. Zrnić, 2005: Polarimetric tornado detection. J. Appl. Meteor., 44, 557-570, CrossRef.

Sireci, O., 2008: Comparison of transmitters of weather radars: Preprints, WMO Technical Conference on Meteorological and Environmental Instruments and Methods of Observation, St. Petersburg, Russia, World Meteorological Organization, P1.29. [Available online at www.wmo.int/pages/prog/www/IMOP/publications/ IOM-96 TECO-2008/P1(29) Sireci Turkey.doc.]

Schultz, C. J., and Coauthors, 2012a: Dual-polarization tornadic debris signatures part I: Examples and utility in an operational setting. Electronic J. Oper. Meteor., 13, $120-137$

Schultz, C. J., and Coauthors, 2012b: Dual-polarization tornadic debris signatures part II: Comparisons and caveats. Electronic J. Oper. Meteor., 13, 138-150.

Thompson, R. L., and Coauthors, 2017: Tornado damage rating probabilities derived from WSR-88D data. Wea. Forecasting, 32, 1509-1528, CrossRef.

Torres, S., and C. Curtis, 2007: Initial implementation of super-resolution data on the NEXRAD network. Preprints, 23rd Int. Conf. on Interactive Information and Processing Systems (IIPS) for Meteorology, Oceanography, and Hydrology, San Antonio, TX, Amer. Meteor. Soc., 5B.10. [Available online at ams.confex. com/ams/pdfpapers/116240.pdf]. 
Van Den Broeke, M. S., and S. T. Jauernic, 2014: Spatial and temporal characteristics of polarimetric tornadic debris signatures. J. Appl. Meteor. Climatol., 53, $2217-$ 2231, CrossRef. 